\title{
Long-term luminosity variations and period changes in CG Cygni`
}

\author{
M. Afşar ${ }^{1}$, P. A. Heckert ${ }^{2}$, and C. İbanoğlu ${ }^{1}$ \\ ${ }^{1}$ Ege University, Science Faculty, Department of Astronomy and Space Sciences, Bornava, Izmir 35100, Turkey \\ e-mail: ibanoglu@astronomy.sci.ege.edu.tr \\ 2 Western Carolina University, Department of Chemistry and Physics, Cullowhee, NC 28723, USA \\ e-mail: heckert@email.wcu.edu
}

Received 27 August 2003 / Accepted 16 February 2004

\begin{abstract}
We report new photometry of CG Cyg from 1998-2002. We also analyze published photometry collected since 1965. An O-C analysis reveals evidence of a third body in the system having a 51 year orbital period. After removing the effects of the third body, we note a remaining 22.5 year cyclic variation in the $\mathrm{O}-\mathrm{C}$ curve that is similar to the period of the luminosity variations. Modeling the spot activity for $28 \mathrm{~V}$ band light curves obtained between 1965 and 2002 confirms the presence of two active longitude belts (ALBs) at approximately $90^{\circ}$ and $270^{\circ}$. Most of the spots occur at mid latitudes with a few at low latitudes. We note no spots at latitudes greater than $60^{\circ}$.
\end{abstract}

Key words. stars: activity - stars: binaries: eclipsing - stars: individual: CG Cyg - stars: starspots

\section{Introduction}

The eclipsing binary CG Cygni (BD $+34^{\circ} 4217$, HIP 103505 , $V=9$. 7, Sp. G9+K3, $P=0.63$ ) is a well-known RS CVn-type star. Among the most peculiar of the short-period chromospherically active binary stars, it is No. 177 in the cata$\log$ of Chromospherically Active Binary Stars (Strassmeier et al. 1993). Since its eclipsing nature was discovered by Williams (1922), many light curves have been obtained by various authors (Yü 1923; Milone et al. 1979; Jassur 1980; Sowell et al. 1987; Naftilan et al. 1987; Bedford et al. 1987; Dapergolas et al. 1988, 1989a,b, 1994; Zeilik et al. 1989, 1991; Kjurkchieva et al. 2003). These workers have shown that the light curve of CG Cyg changes over short and long time intervals. Naftilan et al. (1987) found that the mean light level outside eclipse increased between 1964 and 1976, after which the brightening of the mean light level halted. Sowell et al. (1987) confirmed this result, noting that the mean light level outside eclipse increased by $15 \%$ rather uniformly between 1964 and 1977. A third minimum at phase 0.36 detected by Bedford et al. (1987) was attributed to the circumstellar material located in the orbital plane. Such depressions in the light curve

Send offprint requests to: $\mathrm{M}$. Afşar,

e-mail: afsar@astronomy.sci.ege.edu.tr

* Tables 2, 3 and 5 are only available in electronic form at the CDS via anonymous ftp to cdsarc.u-strasbg.fr (130.79.128.5) or via

http://cdsweb.u-strasbg.fr/cgi-bin/qcat?J/A+A/420/595 have been observed recently by Kjurkchieva et al. (2003) at phases 0.17 and 0.78 .

The orbital period changes of CG Cyg have been the subject of various papers since Milone \& Ziebarth (1974) reported a possible change in the period. The studies on the orbital period changes can be divided into three categories: parabolic fits, multiple linear fits, and a third body in the system. Initial studies assumed that $\mathrm{O}-\mathrm{C}$ values can be fit with a parabola (cf. Milone et al. 1979; Naftilan et al. 1987). Then, Hall (1991) represented the $\mathrm{O}-\mathrm{C}$ values with four straight-line segments, i.e. a decrease and a subsequent increase and so on, in the orbital period. He suggested that the mean brightness of the system outside eclipse and the period vary with the same cycle length by about 50 years. Finally, Albayrak et al. (1999) took into account the possibility of a light-time effect caused by a third body in the system. They fit a model with a third-body orbit of period $46.54 \mathrm{yr}$, an eccentricity of 0.12 and an orbit of the third that is coplanar with the eclipsing pair's orbit. They estimated a mass about $0.12 M_{\odot}$ for the third body.

Naftilan \& Milone (1979) detected a third emission component in the middle of the Ca II $\mathrm{K}$ line. This weak emission, which was best seen on the higher quality plates taken near quadrature, moves not with either star but rather at the systemic velocity. They explain this feature as material concentrated in the $L_{1}$ point or in a shell surrounding both stars. Popper (1994) and Lazaro \& Arevalo (1997) published spectroscopic observations of CG Cyg, but found no evidence for the existence of a third component in the spectra. However, Lazaro \& Arevalo 
found an $\mathrm{H} \alpha$ excess from both components. In addition, they noted that the variations in $\mathrm{H} \alpha$ do not seem to be related to the presence of photometric spots. The most recent spectroscopic observations in the spectral region around the $\mathrm{H} \alpha$ line of CG Cyg were made by Kjurkchieva et al. (2003). They clearly revealed a third absorption line between the spectral profiles of the components both in the $\mathrm{H} \alpha$ and Fe I 6678 lines. The depth of this line changed with the phase the cycle and was comparable to the depths of lines of the component stars. They suggest that this third component originated from cool matter around the center-of-mass of the binary.

The more recent observers and analyzers of the photometry and spectroscopy of CG Cyg have been interested in studying the light curve variations, the spectral line behaviors and the interconnection between them. These studies led to evaluations of starspot parameters and their variations with time, as well as physical parameters of the component stars. The aim of this study is to analyze all the photoelectric times of minima, including new ones obtained by the present authors, and also to analyze all the available light curves to reveal the spot parameters.

\section{Observations}

Johnson BV observations of CG Cygni were obtained on the nights of 23, 24 June and 1, 2 July 2000 and 14, 15, 17, 28 July and 6 August 2001 by an SSP5-type photometer mounted on the $0.5 \mathrm{~m}$ telescope of the Ege University Observatory (EUO, Izmir (Turkey)). The integration time for each band was $10 \mathrm{~s}$. All observations were made in the normal manner, and followed well-known reduction procedures. We obtained differential magnitudes, in the sense of variable minus comparison, and then corrected for atmospheric extinction for each band using the observed magnitudes of the comparison star. We used $\mathrm{BD}+34^{\circ} 4216$ as a comparison star. The data were phased using the ephemeris:

HJD $($ MinI $)=2451522.2441+0.631143144 E$.

Figure 1 presents the $V$-band light curve obtained at the EUO in 2001.

We also obtained new Johnson Cousins BVRI photometry of CG Cyg from the Mount Laguna Observatory in 1998 to 2002. For these observations we used the $0.61 \mathrm{~m}$ telescope equipped with a Hamamatsu R943-02 tube operated at $-1450 \mathrm{~V}$ and thermoelectrically cooled to $-15^{\circ} \mathrm{C}$. The same comparison star was used. Using standards from Landolt (1983) we transformed the magnitudes to the standard system and found average calibrated magnitudes for this comparison star of $B=9.71, V=8.96, R=8.46, I=7.97$, which agree with the values for $B$ and $V$ reported by Milone (1979). Each reported data point in our light curves represents the average of two $20 \mathrm{~s}$ integrations. The light curves are shown in Fig. 2.

From the observations bf obtained between 1999-2002, 25 epochs of minimum light, including 15 primary and 10 secondary eclipse timings, were determined by the Kwee-van Woerden (1956) algorithm using the least squares fit of the data. The new timings of the minimum light together with those published by other authors are listed in the table

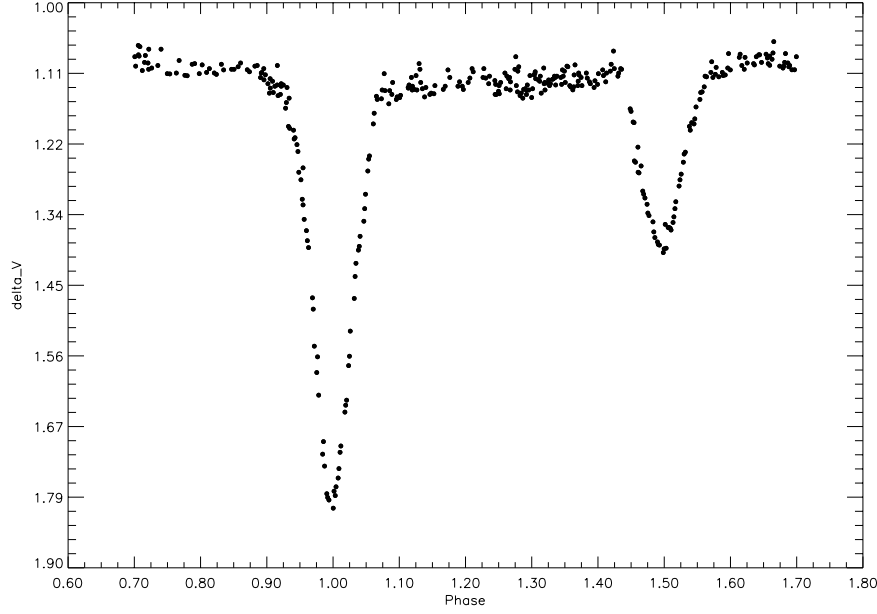

Fig. 1. The $2001 V$-band light curve of CG Cyg (Ege University Observatory).

which is accessible in electronic form from the Strasbourg Astronomical Data Center (CDS).

\section{Eclipse timings and period study}

A possible orbital period change of CG Cyg was first reported by Milone \& Ziebarth (1974). Since the secondary minimum changes are in the same sense as the primary minimum they used both to improve the light elements. They determined two sets of ephemerides for pre-1967 and post-1967, and noted a decrease in the orbital period after 1967. Milone et al. (1979) applied a parabolic fit to the available $\mathrm{O}-\mathrm{C}$ data obtained between 1965 and 1977. They concluded that the orbital period of the system appears to have lengthened and to be continuously changing since the late 1960s. Naftilan et al. (1987) confirmed this finding and suggested a period change of $4.15 \times$ $10^{-6} \mathrm{~s} /$ day.

Hall (1991) updated the O-C curve and suggested that the mean brightness outside eclipse and the orbital period vary with the same cycle length of about $50 \mathrm{yr}$, on the basis of multiple linear fits. This may arise from the magnetic activity of the G9 primary component and it was the first test case for confirmation of Applegate's (1992) prediction. Applegate (1992) proposed that the orbital period changes in RS CVn type binaries are a consequence of magnetic activity in one or both of the component stars. He proposed not only a mechanism that explains period changes, but also suggested a possible connection with the variations of the luminosity of the system. According to his theory, angular momentum exchanges are accompanied by a variation of the total kinetic energy of the star. This energy is supplied by the stellar luminosity. This idea was reexamined and improved by Lanza et al. (1998) and by Lanza \& Rodono (1999), who included the effects of a magnetic field on the internal mass distributions of the active star. This model has been applied to 46 close binary systems and evidence was found that orbital period modulation is related to magnetic activity. Sowell et al. (2001) also investigated the period changes for $\mathrm{CG}$ Cyg and presented an $\mathrm{O}-\mathrm{C}$ variation which is similar to Hall's (1991). The large orbital period change observed 

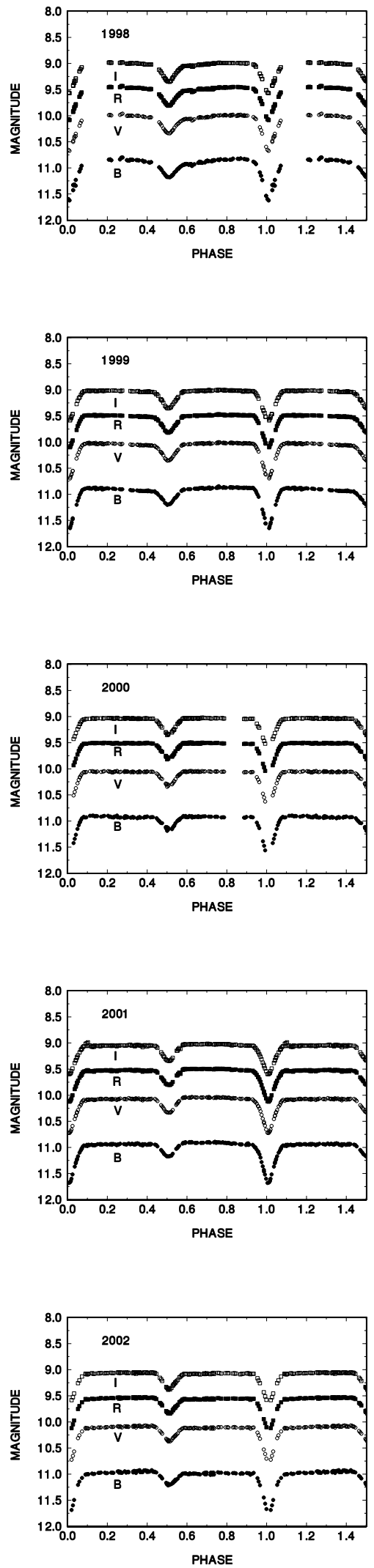

Fig. 2. The 1998-2002 BVRI light curves of CG Cyg (Mount Laguna Observatory).

in this binary was attributed to the chromospheric activity in one or both components of the system. Albayrak et al. (1999) studied long-term period variation of six RS CVn type binaries, including $\mathrm{CG}$ Cyg. They suggested that the most probable cause of the sinusoidal $\mathrm{O}-\mathrm{C}$ variation was the light-time effect due to an invisible component, dynamically bound to the eclipsing pair. Their analysis of $155 \mathrm{O}-\mathrm{C}$ residuals yielded a period of $46.54 \mathrm{yr}$ for the orbital revolution of the eclipsing pair around the center-of-mass of the triple system. A mass of about $0.12 M_{\odot}$ was estimated for the third component assuming the third-body orbit is coplanar with that of the eclipsing pair.

We have obtained 25 timings and combined them with all published epochs of both mid-primary and mid-secondary eclipses as in the table accessible in electronic form. The behavior of the deviation of each timing from the mean orbital period depends strictly on the mean period used at the calculation. Figure 3 shows the $\mathrm{O}-\mathrm{C}(\mathrm{I})$, which is the difference in the values of the primary and secondary minima from the ephemeris given by (Yü 1923),

HJD $($ MinI $)=2422967.4268+0.631139 E$.

The $\mathrm{O}-\mathrm{C}(\mathrm{I})$ deviations indicate a slow decrease and fast increase, i.e. a distorted sine-like variation, with two definite minima and a maximum. Such a sinusoidal variation of $\mathrm{O}-\mathrm{C}$ deviations may be interpreted as having three possible causes: an apsidal motion, magnetic activity in one or both components, or a light-time effect. Because the $\mathrm{O}-\mathrm{C}$ residuals for secondary eclipse are changing in the same direction as that the primary minimum and the orbit of the eclipsing pair is circular, the rotation of the apsis can easily be ruled out. The magnetic cycle of the binary, which will be discussed in the next section, is shorter than the period of sine-like variation. Therefore, we are left with the remaining possibility: the eclipsing pair orbits around the center-of-mass with a third component. Along the line of sight edge-on to the plane, changes in the distance to the observer cause changes in the times of minima, which is known as a "light time effect".

The additional time delay of any observed eclipse due to orbiting around a third body can be represented by (Mayer 1990),

$\Delta T=\frac{A}{\sqrt{1-e^{2} \cos ^{2} \omega}}\left[\frac{1-e^{2}}{1+e \cos \omega} \sin (v+\omega)+e \sin \omega\right]$

where $A$ is the semi-amplitude of the light-time effect, and $v, e$ and $\omega$ are the parameters of the third-body orbit (true anomaly, eccentricity and length of periastron, respectively). In this case the resulting eclipse ephemeris is given by

$T_{\mathrm{ec}}=T_{1}+E P_{1}+\Delta T$

where $T_{1}$ is the starting epoch, $E$ is the integer eclipse cycle number and $P_{1}$ is the orbital period of the eclipsing binary. To obtain the parameters of the third-body orbit, i.e. $P_{2}, T_{2}, e, \omega$ and $a \sin i$ as well as the light elements of the eclipsing pair $T_{1}$ and $P_{1}$, we used a weighted least squares solution. The iterations were continued until the best fit, i.e. the minimum sum of squared standard deviation values was achieved. The results are given in Table 1.

We found the semi-amplitude of the $\mathrm{O}-\mathrm{C}$ curve to be 0.0058 due to the third-body orbit, while it was estimated by 


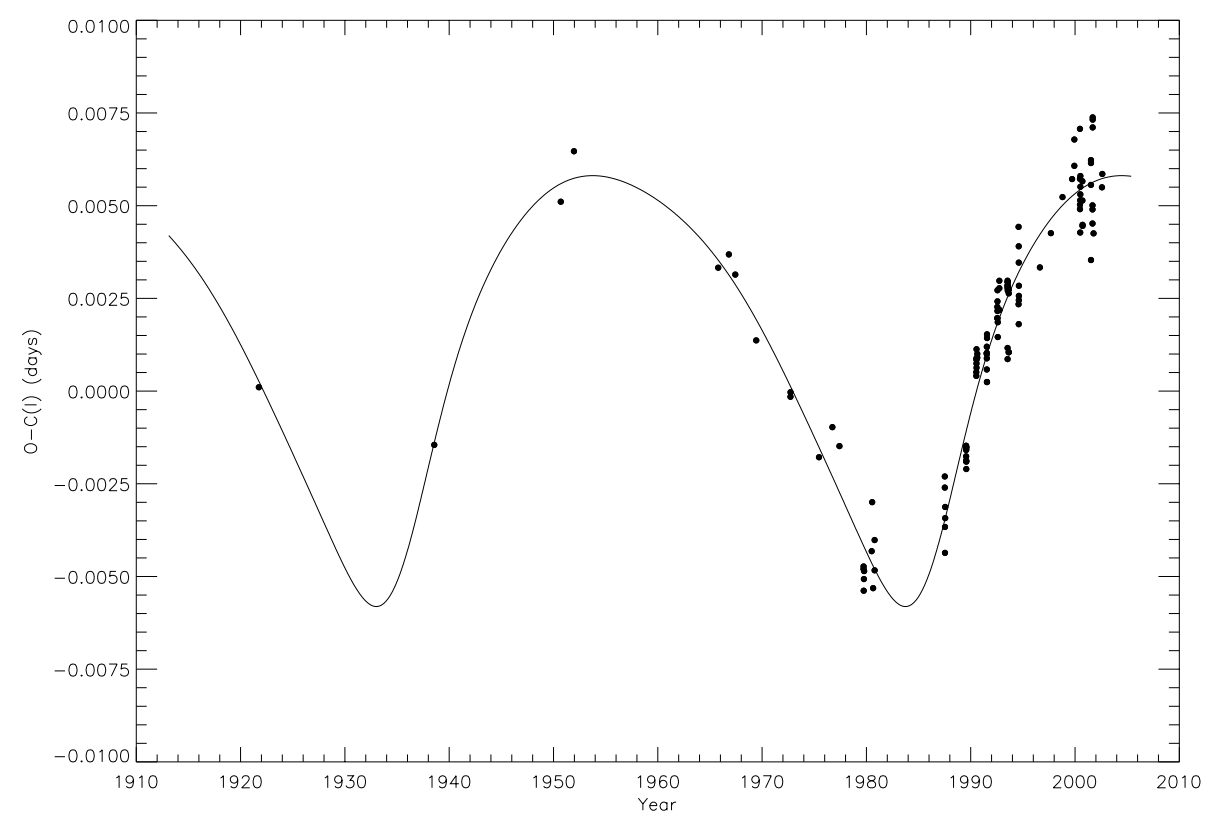

Fig. 3. The O-C(I) curve for CG Cyg based on the linear ephemeris of Yü (1923). The solid line indicates the computed light-time effect. The first four points are photographic times.

Table 1. Parameters of the third-body orbit.

\begin{tabular}{lrr}
\hline \hline Parameter & Value & Standard deviation \\
\hline$a_{12} \sin i(\mathrm{AU})$ & 1.064 & \pm 0.084 \\
$e$ & 0.56 & \pm 0.11 \\
$\omega(\mathrm{deg})$ & 304.444 & \pm 9 \\
$T_{2}$ & 46380 & \pm 396 \\
$P_{2}($ day $)$ & 18529 & \pm 421 \\
$T_{1}$ & 22967.422 & \pm 0.001 \\
$P_{1}($ day $)$ & 0.63114326 & $\pm 2 \times 10^{-8}$ \\
$A$ (day) & 0.0058 & \pm 0.0004 \\
$f(m)\left(M_{\odot}\right)$ & $4.68 \times 10^{-4}$ & $\pm 4.7 \times 10^{-5}$ \\
\hline
\end{tabular}

Albayrak et al. (1999) as 0.0082 days. We derived the semiamplitude of the radial velocity of the eclipsing pair's center of gravity as $0.8 \mathrm{~km} \mathrm{~s}^{-1}$. Using the projected semi-major axis of $1.064 \mathrm{AU}$ and a period of $50.73 \mathrm{yr}$, the mass function for the third star was calculated as $4.68 \times 10^{-4} M_{\odot}$. Since the masses of the components of the eclipsing pair were derived recently by Kjurkchieva et al. (2003) as 0.97 and 0.80 solar masses and the eclipsing pair orbiting around the common center of mass with a period of $50.73 \mathrm{yr}$, the mass of the third star may be obtained from the mass function. The estimated mass of the third-body depends on the inclination of the long-period orbit. We compute the mass of the third star as $0.12,0.14,0.25$ for inclinations of $90^{\circ}, 60^{\circ}$ and $30^{\circ}$, respectively. If we assume that the mass of the third component is $0.9 M_{\odot}$, the inclination of the third-body orbit should be about $10^{\circ}$. Then, the projected semi-major axis $(a \cos i)$ of the third-body around the eclipsing pair is $19 \mathrm{AU}$.
Using the parameters of the third body orbit, the times for each mid-eclipse have been computed and subtracted from the $\mathrm{O}-\mathrm{C}(\mathrm{I})$ values and the new $\mathrm{O}-\mathrm{C}(\mathrm{II})$ values were obtained. On this remaining $\mathrm{O}-\mathrm{C}(\mathrm{II})$ curve (Fig. 4), a cyclic change is still seen. The semi-amplitude of this variation is about $(200 \pm 35) \mathrm{s}$. with a cycle length about $(22.5 \pm 0.7)$ yr. This small amplitude, shorter period change in the orbital period of the eclipsing pair may be attributed to the magnetic activity, because brightness variation of the system has a similar cycle length (see next section). The time differences between observed and calculated by light-time orbit and sine-like change have also been plotted in Fig. 5.

\section{The variation of the mean brightness}

Many complete light curves of CG Cyg have been obtained and published by various authors since 1921. In general, two comparison stars were used in the photometric observations. Most of the observers used $\mathrm{BD}+34^{\circ} 4216$ as a comparison while the others preferred either Yü's (a) (GSC 02696-02622) or (b) (GSC 02696-02207) stars as the comparison. To reduce all the light curves to a common base we determined the brightness differences between the primary comparison star, BD $+34^{\circ} 4216$ and the others. We observed these stars near the meridian on the same night and derived the magnitude difference in $B$ and $V$ bands, BD $+34^{\circ} 4216$ minus Yü's stars, as $\Delta B=-1.083 \pm 0.018$ and $\Delta V=-1.480 \pm 0.019$ for (a) and $\Delta B=-1.341 \pm 0.019$ and $\Delta V=-1.610 \pm 0.011$ for $(\mathrm{b})$ respectively. The magnitude differences between Yü's (b) and Yü's (a) stars are 0.258 and 0.130 in $B$ and $V$ bands. These differences were also derived by Jassur (1980) as 0.279 and 0. 114 for the corresponding bandpasses. Standard magnitudes for $\mathrm{BD}+34^{\circ} 4216$ were given by Milone et al. (1979) as $V=$ 8.969, $B=9$ m.713. Using the differences we reduced all the light curves to the standard system. 


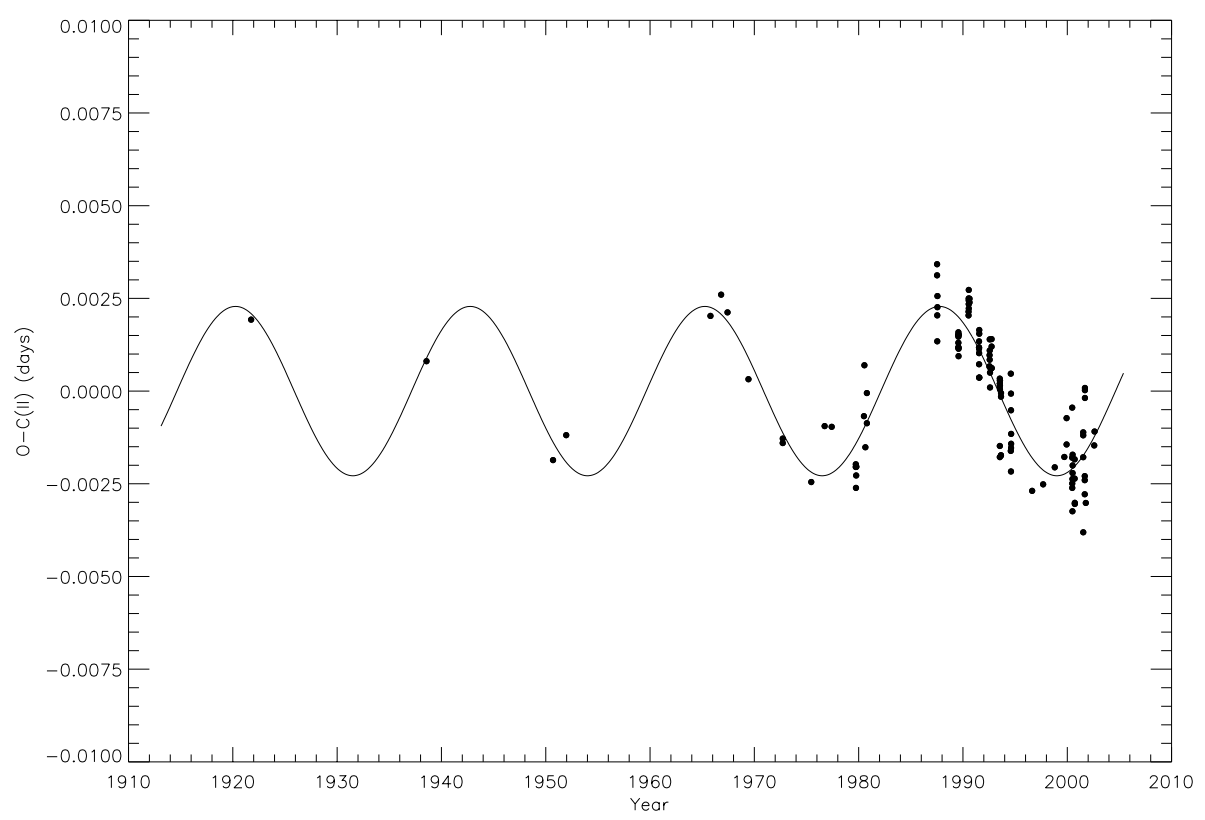

Fig. 4. An O-C comparison between eclipse timings and the third-body ephemeris and the remaining cyclic changes.

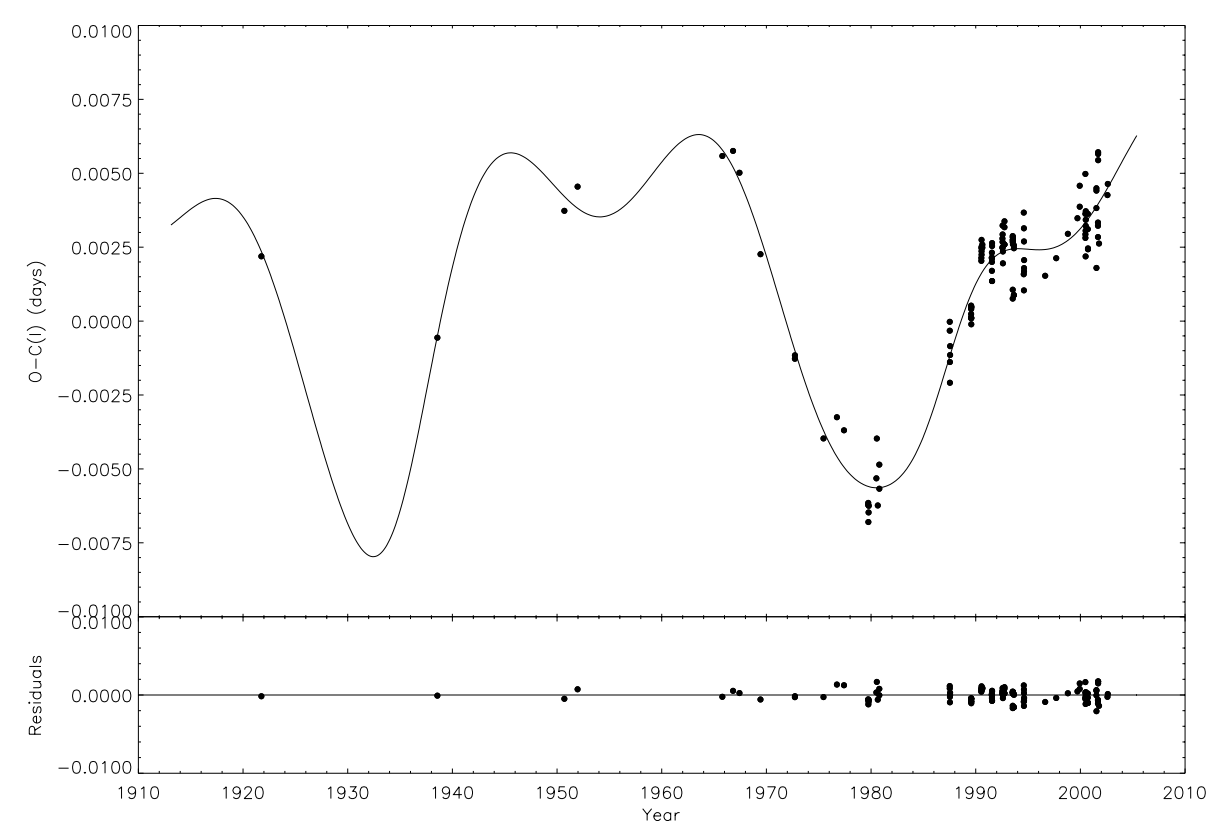

Fig. 5. Light-time values and sine-like variation due to another cause (attributed to the magnetic activity) and the computed O-C values. The residuals of $\mathrm{O}-\mathrm{C}$ values from the computed curve are shown in the bottom panel.

After transforming all the available differential observations into the standard system, the magnitudes were read from the each light curve at four phases $0.0,0.25,0.5,0.75$. These data, presented in Table 2, are plotted in Fig. 6 and cover the time interval between 1965 and 2002. The data gathered between 1992 and 2002 show that the mean $B-V$ color of the system outside eclipse gets redder by about 0.03 mag when the brightness dims. The brightness and color appear to change in the same direction as previously predicted by Hall (1991). As the $\mathrm{O}-\mathrm{C}$ residuals are decreasing the brightness dims and the $B-V$ color gets redder.

The variations of the mean brightness at mid-primary eclipse show an almost cyclic change in both the $B$ and
$V$ bandpasses. The amplitude of the variation is about $0.15 \mathrm{mag}$ in $B$ and 0.13 mag in the $V$ band. While the mean brightness was the smallest around 1967 for these four phases it reached the maximum around 1978. The brightness began to decrease until 1986 then increased again. Both the two maxima and the two minima, have different values. The pattern of the variations is suggestive of either the existence of an approximately 20-year period with a changing amplitude or two kinds of variations with different amplitudes having a 40-year period.

In Figs. $6 \mathrm{~b}$ and $6 \mathrm{~d}$ we show the variation of the brightness at the first and second maxima. The amplitude of the variation of the brightness at first maximum is about $0.17 \mathrm{mag}$ in $B$ and 0.14 mag in $V$ band. The brightness seems to change with a 

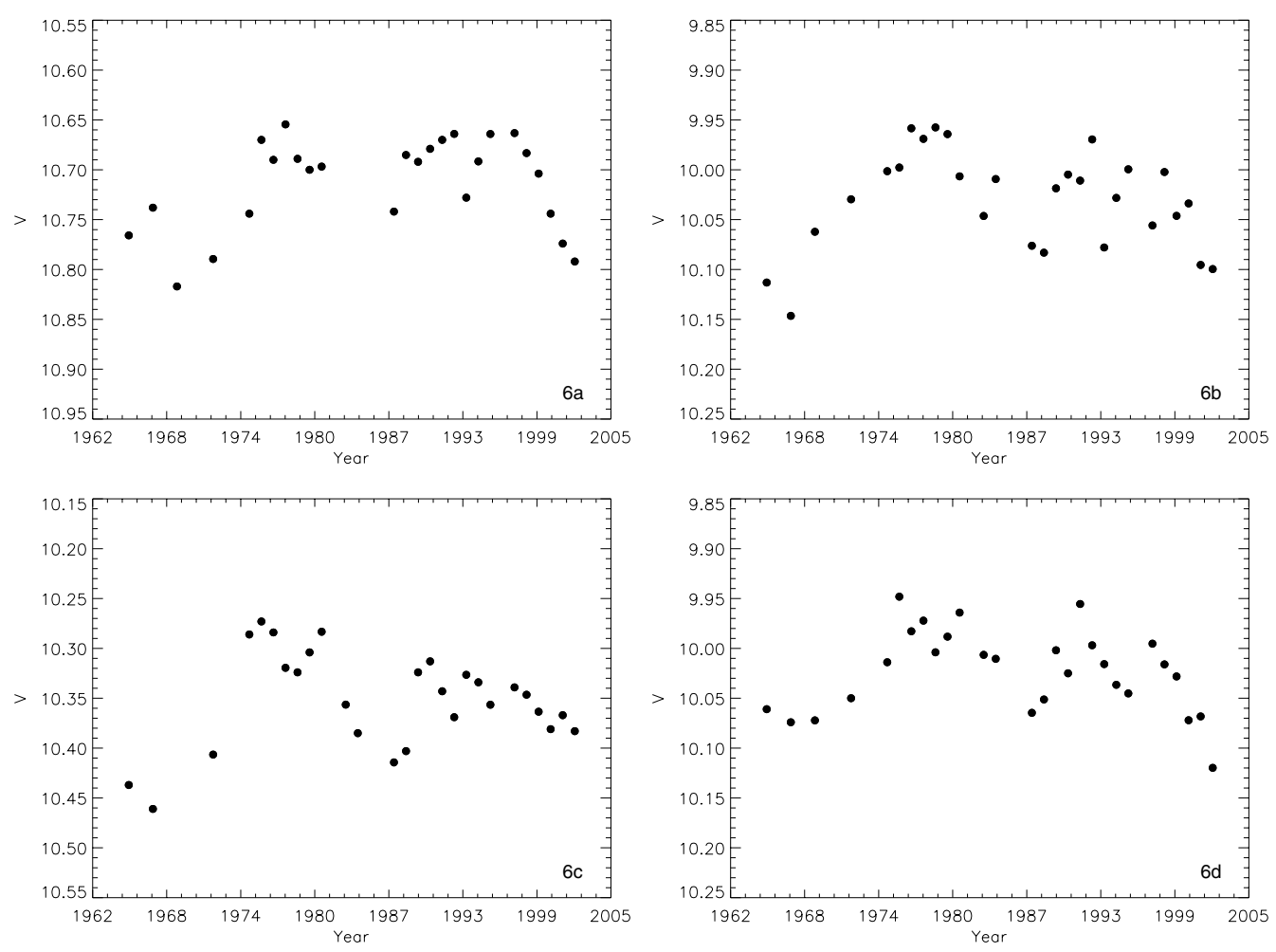

Fig. 6. Standard $V$ magnitudes for CG Cyg. a) At phase 0.0 , b) 0.25 , c) 0.5 and d) 0.75 , respectively.

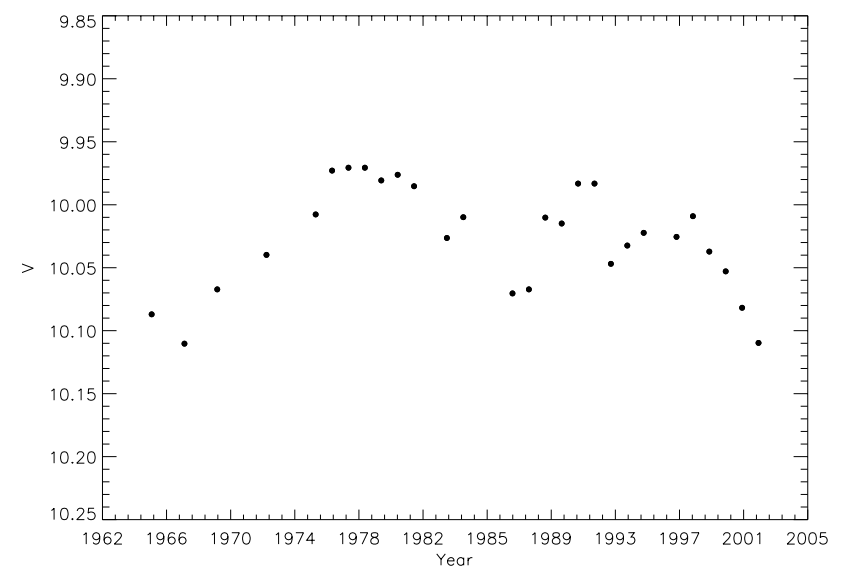

Fig. 7. Variation of the mean brightness level at maxima, i.e. $($ MaxI + MaxII)/2.

period of about 21 years. The amplitude in the color variation is less than $0.03 \mathrm{mag}$. The variation of the mean brightness at second quadrature seems to be smaller than that of first maximum. Figure 7 shows the variation of the mean brightness level at maxima, i.e. (MaxI + MaxII)/2, at different epochs. This figure also shows that the variation in mean brightness at quadratures is almost cyclic but not exactly periodic, because the time intervals between consecutive minima and maxima are not equal. The data obtained over 37 years indicate that the mean brightness of the system has reached, at least, two local maxima and two minima. Despite the large fluctuations in magnitudes the average period seems to be about $18 \mathrm{yr}$. It should also be noted that the magnitude difference between the maxima, in the sense of MaxI - MaxII, varies with almost the same period.
In addition to these long term brightness variations, previous observers have noted short term fluctuations relative to the orbital period. These fluctuations were initially attributed to the variability of the comparison star, which was Yü's star (a). Zeilik et al. (1991) and Beckert et al. (1989, 1991) used Yü's (a) star as the comparison. However, Heckert \& Zeilik (1991) observed this star continuously for about $1.5 \mathrm{~h}$ and found a variation of only about $0.04 \mathrm{mag}$ in its brightness which was not enough to explain the light fluctuations in CG Cyg. In addition, Dapergolas et al. (1991) observed such fluctuations using $\mathrm{BD}+34^{\circ} 4216$ as the comparison star. Other observations show that these fluctuations in CG Cyg do not occur at all epochs. Hence, Zeilik et al. (1994) concluded that the observed light fluctuations do not originate in the comparison star used, but that the rapid, small amplitude fluctuations are intrinsic to the system.

\section{Analysis of the light curves}

Zeilik et al. (1994) collected all the available photometric data from 1922 to 1991 and analysed for the orbital parameters of the system, as well as the starspot parameters. They found that the spots on CG Cyg tend to be located at two active longitude belts (ALB) at about $90^{\circ}$ and $270^{\circ}$, i.e. at quadrature longitudes. Photometric data obtained after 1991 have been analyzed by Heckert (1994, 1996, 1998), Dapergolas et al. (2000) and Kjurkchieva (2003). They found similar results in that the wave-like distortions on the light curves could be represented by two cool spots on the primary component. In addition to wave-like distortion on the light curves, Bedford et al. (1987), 


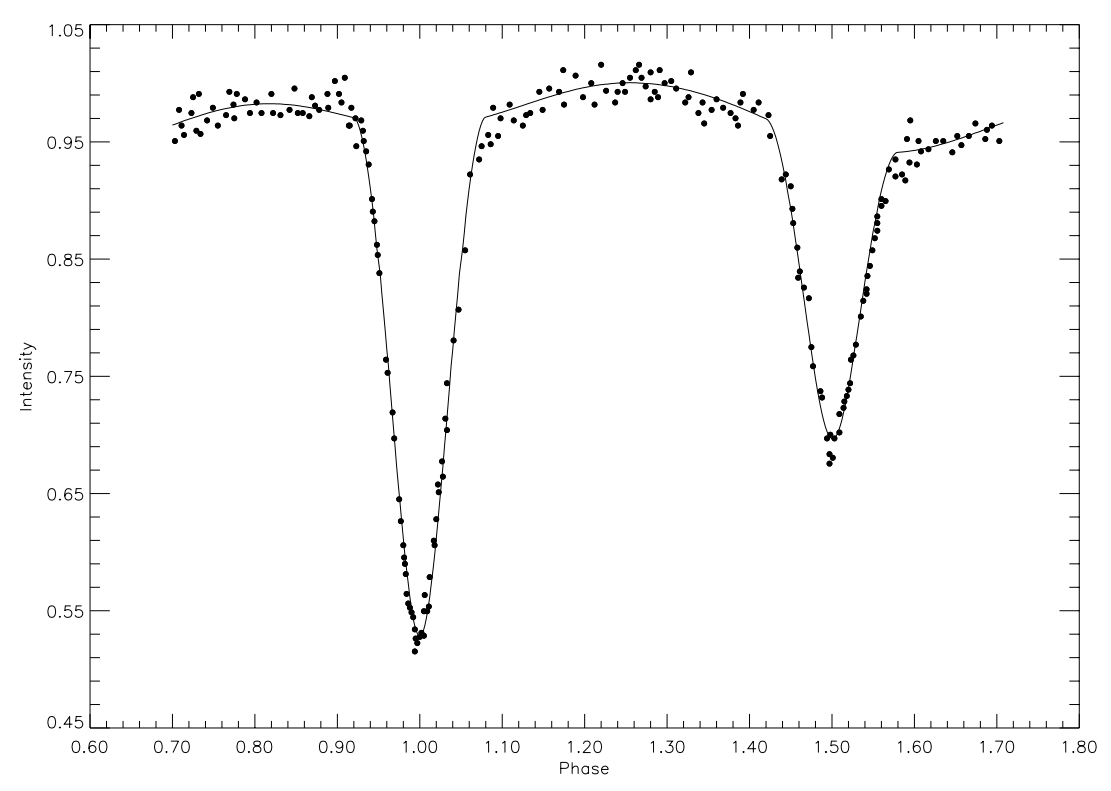

Fig. 8. The $1992 V$-light curve and the computed curve by using the WD code.

Zeilik et al. (1994) and Kjurkchieva (2003) detected some depression on the light curves at different orbital phases.

We collected photometric data for as many complete light curves as possible from the literature beginning in 1965. A total of 28 light curves obtained between 1965 and 2002, listed in Table 3, were analyzed by the Information Limit Optimization Technique (ILOT) described by Budding \& Zeilik (1987) and Banks (1989). The main assumptions made during the analysis by the ILOT are: (1) the activity originates in the hotter star (late G), (2) maculation effects are separable from proximity and eclipse effects, and (3) a cool, circular spot model may represent the parameters of the spotted regions, i.e. longitude, latitude, radius and temperature relative to the photosphere. The reliability of these key parameters of the stellar magnetic activity, of course, depends on the photometric accuracies. Due to the small light contribution of the secondary star, which amounts to 0.34 in the $V$ bandpass, the hotter primary star should be responsible for the magnetic activity. Therefore we assumed that the primary component should be heavily spotted.

The steps of our analysis are the same as those used by Zeilik et al. (1994): (1) optimize a model eclipsing binary curve to the observations; (2) calculate the residuals by subtracting data points from the model to define the maculation wave; (3) optimize a model for the maculation wave to calculate the starspot parameters.

First, we solved the 1992 V-light curve of CG Cyg to obtain the input parameters. This light curve, which is complete, has a small amplitude wave-like distortion located just after secondary eclipse. The one-spot solution with the standard package of the Wilson-Devinney code (Wilson \& Devinney 1971; Wilson 1993) gives the parameters listed in Table 4. The final fit is compared with the observations in Fig. 8. The second
Table 4. $1992 V$-light curve solutions.

\begin{tabular}{lc}
\hline \hline Parameter & Value \\
\hline$i$ & $82.602^{\circ} \pm 0.099$ \\
$T_{1}{ }^{*}$ & $5260 \mathrm{~K}$ \\
$T_{2}$ & $4714 \pm 10 \mathrm{~K}$ \\
$l_{1}$ & $0.648 \pm 0.010$ \\
$l_{2}$ & $0.337 \pm 0.015$ \\
$r_{1}$ (mean) & $0.250 \pm 0.001$ \\
$r_{2}$ (mean) & $0.241 \pm 0.001$ \\
\hline${ }^{*}$ Popper (1994).
\end{tabular}

step was to produce the distortion curve using ILOT. The difference between the observed curve and the synthetic curve computed using the parameters given in Table 4 was revealed for $28 \mathrm{~V}$-band light curves obtained between 1965 and 2002. The shape of the distortion curve depends on the number of spots, locations and sizes on the star's surface. The distortion curves could be reproduced by two cool spots on the primary star except for 1965, 1967, 1991 and 1992 for which one spot was used. In Table 5 the longitude, latitude and radius of the $\operatorname{spot}(\mathrm{s})$ are listed in degrees for each light curve. The longitude is defined so that $0^{\circ}$ is at the nominal center of primary eclipse and increases with increasing phase. The latitude is defined with $0^{\circ}$ at the equator.

Many of the $V$ light curves that we model here have been previously modeled (Zeilik et al. 1994; Heckert 1994, 1996, 1998) using the same ILOT method. Our new results are generally close to but not exactly the same as the previous results. One source of the small differences in modeling results may be slightly different initial input solutions. The input parameters, derived from the 1992 light curve used in this work 

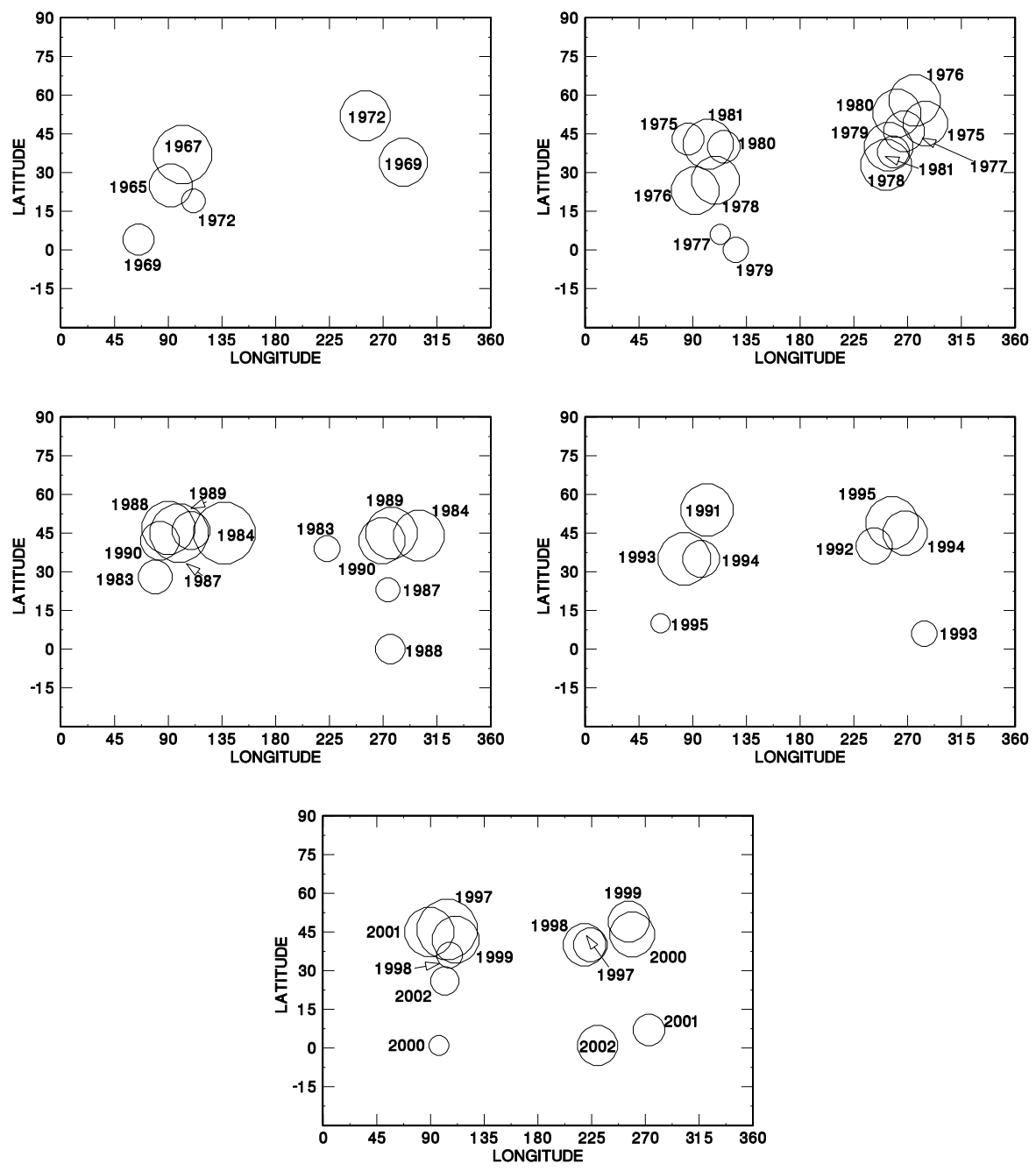

Fig. 9. Mercator maps of the surface of the hotter primary.

differ by a small amount from the parameters used in the previous work. Previous workers obtained the orbital input parameters individually for each year. The 1972, 1975, 1979, 1980, 1981, 1983, 1984, 1987, 1989, and 1995 light curves were previously modeled with just one spot. In this work, we model each of these light curves with two spots. Fitting an additional spot will have an effect on the fit parameters of the existing spot, however the spot parameters usually agree to within the errors. The 1967, 1991, 1994, and 1998 light curves are modeled with the same number of spots as the previous work and tend to agree to within the errors. With the exception of the 1967 and the 1984 light curves, the new results have lower chi square values than the previous results.

We plot the starspot parameters on the Mercator maps of the surface of the hotter primary in Fig. 9. These are the two-dimensional maps of determinate starspot solutions for 1965-2002 for $V$ band only. The size of each circle shows the relative area of spotted regions, labeled by year of observation. It is clearly seen that there is an indication of two active regions on the primary star, which are located in two longitude zones centered around the quadrature points as reported by Zeilik et al. (1994). One is located near $90^{\circ}$ and the other near $270^{\circ}$ (Fig. 10); both are at latitudes between $0^{\circ}$ and $60^{\circ}$.

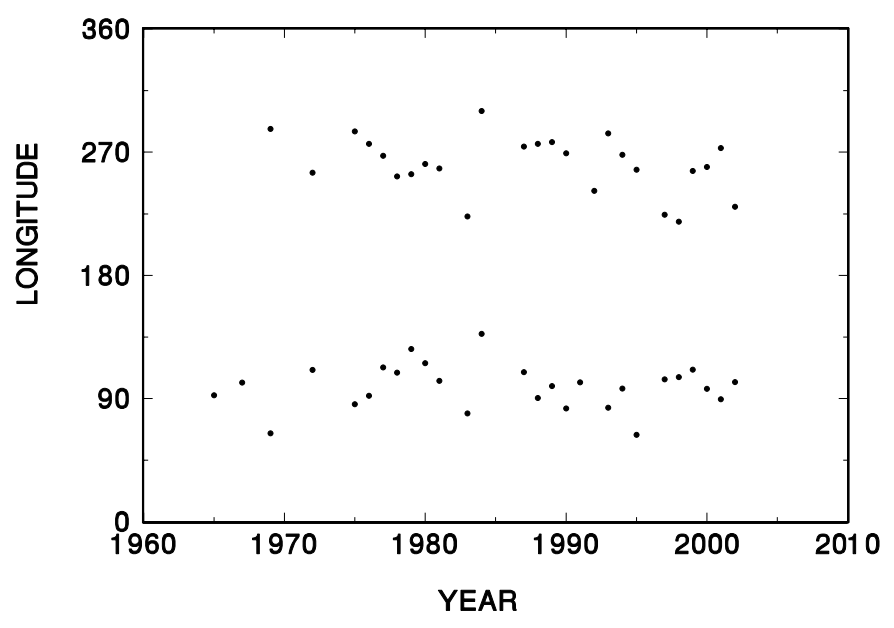

Fig. 10. The changes of the spot longitudes vs. time.

These appear to be the ALBs proposed by Eaton \& Hall (1970) and Uchida \& Sakurai $(1983,1985)$ for RS CVn systems. Such ALBs are found on the Sun and often appear as two regions, tens of degrees wide, separated by roughly 180 degrees (Sawyer 1968). As seen in Fig. 10, the spot longitudes show 
a tendency to migrate in opposite directions. When the longitude in one ALB increases, the other decreases. Some data sets have large observational scatter (for instance, 1965, 1972, 1984) therefore spot parameters for such sets are not reliable. On the other hand some light curves (i.e. 1967, 1969, 1977, $1983,1984,1998$ ) have too large gaps to find a reliable starspot solution. If we do not take into account starspot parameters found from these incomplete and/or less accurate observational data CG Cyg has, in general, two ALBs. While the longitude of spots in the first ALB is decreasing the longitude of spots in the other ALB is increasing.

\section{Conclusions}

For more than a century it has been known that long-term variations occur in the surface activity of our star, the Sun. The dominant one is the well-known 11-year cycle. Chromospherically active late-type stars also display solar-type activity phenomena but to a much stronger degree than the Sun itself. The outstanding photometric indicator of chromospherically-active close binaries is the light variability with an amplitude of about a tenth of a magnitude in phase with the rotation of the active component(s). On the other hand, the photometric signature of a magnetic cycle may be long-term changes in the mean brightness of these systems. Long-term magnetic cycles have been determined to date for only limited number of systems. The length of magnetic cycles for RS CVn type active systems range from about ten years to about a century. Hall (1991) proposed that cyclic changes in mean brightness were common among known chromospherically active stars. The amplitudes of the changes in mean brightness range from about 0.1 to $0.5 \mathrm{mag}$, whereas the luminosity change of the Sun is about $0.001 \mathrm{mag}$ in phase with its 11-year cycle. The enhanced magnetic activity in CG Cyg and similar systems enhances these luminosity variations as well as the spot activity, suggesting that these luminosity variations are also magnetic in nature.

CG Cyg consists of two late-type stars. The G9 primary has an active chromosphere as proven by spectroscopic and photometric studies. However, the latest spectroscopic study made by Kjurkchieva et al. (2003) revealed that the emission cores of the $\mathrm{H} \alpha$ line of both components vary with the orbital phase indicating that both components have inhomogeneous active chromospheres. In addition they have noted that the $\mathrm{H} \alpha$ emmision line of the secondary star showed a flare-like event. The amplitude of the light variations due to spots on the components is at most $0.089 \mathrm{mag}$. Since the light contribution of the primary exceeds 0.6 of the total light of the system, it should be more active than its companion. As is suggested by all previous studies, our light curve analysis indicates two active regions on the more massive primary star.

The brightness variation at four special orbital phases has been studied as well as the total brightness of the system at the maxima. Although the lengths of the time intervals are not the same, the average cycle of the mean brightness is about $20 \mathrm{yr}$. The photometric data obtained over the period of 37 years indicate that at least two definite maxima and minima have been obtained. The largest amplitude of the variation in the mean brightness is about $0.17 \mathrm{mag}$ in $B$ and $0.14 \mathrm{mag}$ in $V$ band.

In addition to the light curve analysis and examination of the mean brightness changes we also studied the orbital period changes. The up-to-date $\mathrm{O}-\mathrm{C}$ deviations resemble the lighttime effect, orbiting around a third body. Therefore we analyzed all available $\mathrm{O}-\mathrm{C}$ data under the hypothesis of a lighttime effect. This analysis yields that the eclipsing pair revolves around the common center of gravity with a tertiary body with a period of about 51 years. The eccentricity of this orbit is about 0.56 , and $a_{12} \sin i=1.06 \mathrm{AU}$. We estimate the mass of the tertiary component as $0.12 M_{\odot}$ for the orbital inclination of the eclipsing pair's orbit. Using the parameters given in Table 2 the times for each mid-eclipse have been computed from the observed ones. The resultant $\mathrm{O}-\mathrm{C}$ values indicate a cyclic change as shown in Fig. 4. The semi-amplitude of these variations is about $200 \mathrm{~s}$ with a cycle length about $22.5 \mathrm{yr}$. This change in the orbital period of the system has been attributed to the magnetic activity in one or both components of CG Cyg. By comparing Figs. 4 and 7 we conclude that the $\mathrm{O}-\mathrm{C}$ curve minimum coincides with the maximum total brightness and vice versa; both have similar period of about 20 years.

Acknowledgements. M. Afşar would like to thank Turkish Scientific and Research Council for their invaluable supports during her Master of Science and Ph.D. studies. P. Heckert thanks Ron Angione and Paul Etzel for scheduling generous amounts of telescope time at Mount Laguna Observatory. Also support from the American Astronomical Society Small Grants Program is acknowledged.

\section{References}

Afşar, M., \& İbanoğlu, C. 2000, IBVS, 4979

Albayrak, B., Özeren, F., Ekmekçi, F., \& Demircan, O. 1999, Rev. Mex. Astron. Astrofis., 35, 3

Applegate, J. H. 1992, ApJ, 385, 621

Banks, T. S. 1989, Masters Thesis, Victoria Univ., Wellington

Beckert, D., Cox, D., Gordon, S., Ledlow, M., \& Zeilik, M. 1989, IBVS, 3398

Beckert, D., Gordon, S., Jaderlund, E., Mann, E., \& Zeilik, M. 1991, IBVS, 3556

Bedford, D. K., Fuensalida, J. J., \& Arevalo, M. J. 1987, A\&A, 182, 264

Budding, E., \& Zeilik, M. 1987, ApJ, 319, 827

Dapergolas, A., Kontizas, E., \& Kontizas, M. 1988, IBVS, 3249

Dapergolas, A., Kontizas, E., \& Kontizas, M. 1989a, IBVS, 3322

Dapergolas, A., Kontizas, E., \& Kontizas, M. 1989b, IBVS, 3380

Dapergolas, A., Kontizas, E., \& Kontizas, M. 1991, IBVS, 3609

Dapergolas, A., Kontizas, E., \& Kontizas, M. 1994, IBVS, 4051

Dapergolas, A., Kontizas, E., \& Kontizas, M. 2000, IBVS, 5011

Eaton, J. A., \& Hall, D. S. 1979, ApJ, 227, 907

Hall, D. S. 1991, ApJ, 380, L85

Heckert, P. A. 1994, IBVS, 4127

Heckert, P. A. 1996, IBVS, 4371

Heckert, P. A. 1998, IBVS, 4627

Heckert, P. A., \& Zeilik, M. 1991, IBVS, 3688

Jassur, M. Z. D. 1980, Ap\&SS, 67, 19

Kjurkchieva, D. P., Marchev, D. V., \& Ogloza, W. 2003, A\&A, 400, 623

Kwee, K. K., \& van Woerden, H. 1956, Bull. Astron. Inst. Netherlands, 12, 327 
Landolt, A. U. 1983, AJ, 88, 439

Lanza, A. F., \& Rodono, M. 1999, A\&A, 349, 887

Lanza, A. F., Rodono, M., \& Rosner, R. 1998, MNRAS, 296, 893

Lázaro, C., \& Arévalo, M. J. 1997, AJ, 1698, 2283

Mayer, P. 1990, BAICz, 41, 231

Milone, E. F., Castle, K. G., Robb, R. M., et al. 1979, AJ, 84, 417

Milone, E. F., \& Ziebarth, K. E. 1974, PASP, 86, 684

Naftilan, S. A., Grillmair, C., \& Trager, G. 1987, AJ, 93, 210

Naftilan, S. A., \& Milone, E. F. 1979, ApJ, 84, 1218

Popper, D. M. 1994, AJ, 108, 1091

Sawyer, C. 1968, ARA\&A, 6, 115

Sowell, J. R., Hughes, S. B., Hall, D. S., \& Howard, B. A. 2001, AJ, 122, 1965

Sowell, J. R., Wilson, J. W., Hall, D. S., \& Peyman, P. E. 1987, PASP, 99, 407

Strassmeier, K. G., Hall, D. S., Fekel, F. C., \& Scheck, M. 1993, A\&AS, 100, 173
Uchida, Y., \& Sakurai, T. 1983, in Activity in red-dwarf stars, ed. P. B Byrne, \& M. Rodono (Dordrecht: Reidel), in IAU Coll., 71, 629

Uchida, Y., \& Sakurai, T. 1985, in Unstable current systems and plasma instabilities in astrophysics, ed. M. R. Kundu, \& G. D. Holman (Dordrecht: Reidel), in IAU 107th Symp., 281

Williams, A. S. 1922, MNRAS, 82, 300

Wilson, R. E. 1993, New Frontiers in Binary Star Research, ed. J. C. Leung, \& I.-S. Nha, in ASP Conf. Ser., 38, 91

Wilson, R. E., \& Devinney, E. J. 1971, ApJ, 166, 605

Yü, C.-S. 1923, ApJ, 58, 75

Zeilik, M., Cox, D. A., De Blasi, C., Rhodes, M., \& Budding, E. 1989, ApJ, 345, 991

Zeilik, M., Elston, R., Henson, G., Schmalke, P., \& Smith, P. 1982, IBVS, 2138

Zeilik, M., Gordon, S., Jaderlund, E., et al. 1994, ApJ, 421, 303

Zeilik, M., Heckert, P. A., Ledlow, M., et al. 1991, IBVS, 3663 